\title{
Monitoring the Red Palm Weevil Infestation Using Machine Learning and Optical Sensing
}

\author{
Yuan Mao ${ }^{1, \hbar}$, Islam Ashry ${ }^{1, \hbar}$, Biwei Wang ${ }^{1,2, \hbar}$, Yousef Al-Fehaid ${ }^{3}$, Abdulmoneim \\ Al-Shawaf ${ }^{3}$, Tien Khee $\mathrm{Ng}^{1}$, Changyuan $\mathrm{Yu}^{2}$, and Boon S. Ooi ${ }^{1, *}$ \\ ${ }^{1}$ Computer, Electrical and Mathematical Sciences and Engineering (CEMSE) division, King Abdullah University \\ of Science and Technology (KAUST), Thuwal, 23955-6900, Kingdom of Saudi Arabia \\ ${ }^{2}$ Department of Electronic and Information Engineering, The Hong Kong Polytechnic University, Hong Kong \\ SAR, China \\ ${ }^{3}$ Center of Date Palms and Dates, Ministry of Environment, Water and Agriculture, Al-Hassa, Kingdom of Saudi \\ Arabia \\ ${ }^{\ddagger}$ These authors contributed equally to this work \\ *boon.ooi@kaust.edu.sa
}

\begin{abstract}
Red palm weevil (RPW) has destroyed many palm farms worldwide. Early detection of RPW is challenging. We introduce combining machine learning and fiber optic distributed acoustic sensing for detecting the RPW in its larvae stage. (C) 2021 The Author(s)
\end{abstract}

\section{Introduction}

The date, coconut, and oil palms are important nutritious and economical crops that provide healthy food to millions of people worldwide. Unfortunately, these crops are vulnerable because they have been destroyed by the red palm weevil (RPW) [1]. RPW is the world's most detrimental pest of palm trees. This plague has been found in more than 60 countries [2] and results in wiping out many palm farms causing serious economic losses. Healing infested palm trees is feasible; however, detecting the RPW infestation in an early stage is challenging, especially in large-scale farms. Visual inspection of palms is a reliable technique for detecting the RPW; however, it is ineffective because an infested palm tree shows visual distress signs too late, when it is difficult to rescue the tree. X-ray based tomography [3] and trained dogs [4] are effective methods for the early detection of RPW; however, they lack feasibility in vast farms because of their slow scanning process.

As we have recently reported in [5], a fiber optic distributed acoustic sensor (DAS) can detect the weevil larvae sound in an early stage (first two weeks of the weevil larvae stage). Given the distributed sensing ability of the DAS, a single fiber optic cable can simultaneously monitor hundreds of trees. Since our former work was carried out in a controlled-environment, a straightforward signal processing algorithm was adopted to recognize the larvae sound from the background noise. Deploying an optical fiber in open-air farms would result in having wind, bird sounds, etc., as acoustic noise sources, which would require a powerful events classification method to recognize the larvae sound from the other noises. Here, we introduce using a convolutional neural network (CNN) to identify healthy and infested trees, based on the data collected by the fiber optic DAS. Within the laboratory environment, we mimic a farm's environment by having healthy/infested tree, wind, and birds sound. This work is an important preamble towards combining machine learning and fiber optic DAS for the early detection of RPW in real farms.

\section{Experimental setup}

The overall design of our experiment is shown schematically in Fig. 1. The fiber optic DAS, designed using the phase-sensitive optical time-domain reflectometry ( $\phi$-OTDR) [6], comprised a narrow linewidth laser that produced a continuous wave $(\mathrm{CW})$ light. Using an acousto-optic modulator (AOM), the CW light was modulated into pulses of a $5-\mathrm{kHz}$ repetition rate and a 50-ns width. Throughout a circulator, the pulses were optically amplified with an erbium-doped fiber amplifier (EDFA) and injected into a single-mode fiber (SMF) of a $\sim 2-\mathrm{km}$ length. At a 990-m distance from the input port of the SMF, we wound a 5-m section of the fiber around a tree trunk. The backscattered Rayleigh signal from the SMF was amplified with another EDFA and its amplified spontaneous emission (ASE) noise was filtered out using a fiber Bragg grating (FBG). The filtered Rayleigh signal was then detected by a photodetector (PD) and sampled with a digitizer of a 200-MHz sampling rate and a 1-s recording period. Inside the core of the tree trunk, we implanted a loudspeaker to play the eating sound of a $\sim 12$-days old larvae. Meanwhile, at a $\sim 1-\mathrm{m}$ distance from the tree, we placed a fan to blow air in the direction of the fiber and tree, and another loudspeaker to continuously generate the bird sounds. 


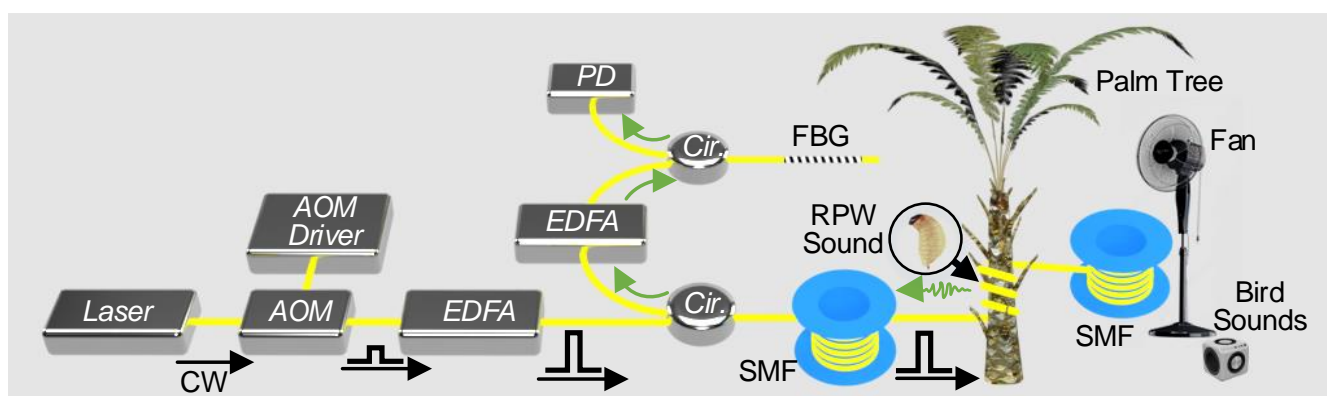

Fig. 1. Experimental setup of using the fiber optic DAS to detect the RPW sound. Cir., circulator.

\section{Results and Discussion}

We initially investigated the temporal and spectral signals of the various acoustic sources included in the experiment. Firstly, we only turned on the loudspeaker that produced the larvae sound inside the tree trunk, while switching off the fan and external loudspeaker. Applying the normalized differential method [6] and fast Fourier transform (FFT) to the Rayleigh signal at the tree position, Fig. 2(a) shows a representative example of the larvae sound's temporal signal. In contrast, Fig. 2(b) shows an example to the wind's temporal signal, recorded when only the fan was turned on while the two loudspeakers were off. Clearly, the tree swinging by the wind has lower vibration frequencies than those caused by the larvae sound [Fig. 2(c)]. On the other hand, the optical fiber did not "hear" the bird sounds produced by the external loudspeaker, when the fan and larvae sound were off. This is to be expected because the air significantly attenuates the energy of the bird sounds that propagates from the loudspeaker to the optical fiber, and also it is difficult for the thin optical fiber to be impacted by the acoustic signal that spatially diverges in air.
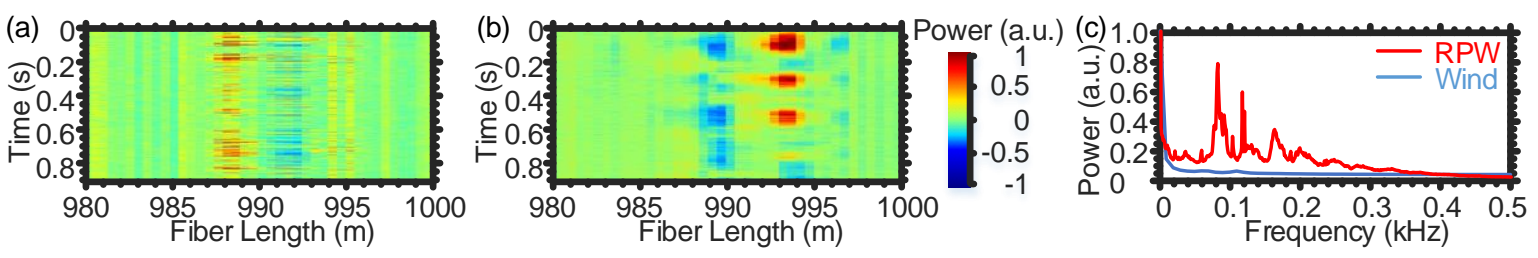

Fig. 2. The temporal signal of the larvae sound (a) and wind (b). (c) The corresponding power spectra of the larvae sound and wind.

Given the features differences between the larvae sound and noises [Fig. 2], CNNs trained through supervised learning [7] could be effective for distinguishing infested and healthy trees in a noisy environment. For such purpose, we arranged the temporal data in a two-dimensional matrix form, resembling those shown in Figs. 2(a) and 2(b). In particular, for the CNN, a training example was $10 \times 5000$ matrix, i.e., 10 spatial points of the 5-m fiber section wound around the tree and 5000 temporal readings along the 1-s recording period of the digitizer. We labeled the data based on the signal-to-noise ratio (SNR) value of the vibration event at the tree position. Regarding the infested tree, the temporal signal was labeled as "infested" when the SNR $>2 \mathrm{~dB}$. For the infested tree, we collected 4,000 examples at various intensity levels for the larvae sound and with/without the wind. In contrast, the signals collected when larvae loudspeaker was turned off were labeled as "healthy", regardless of the SNR value. Similarly, we collected 4,000 "healthy" examples gathered with/without the wind. The entire 8,000 examples were divided as $60 \%$ training, $20 \%$ validation, and $20 \%$ testing datasets, for the purpose of training and evaluating the performance of the $\mathrm{CNN}$.

The CNN's architecture consisted of an input layer, two pairs of convolutional and max pooling layers, a flatten layer, a fully-connected layer, and an output layer [Fig. 3(a)]. Fig. 3(b) shows the evolution of the training/validation accuracy and loss with the epoch. The training cycles exhibit a perfect matching between the training and validation accuracy/loss curves. At the end of the training cycles, a validation accuracy value of $99.4 \%$ is obtained. Using the testing dataset, Fig. 3(c) provides the confusion matrix of the CNN. The performance of the CNN with the temporal data is excellent with a $99.3 \%$ classification accuracy.

To thoroughly understand how the CNN model looks like after the training cycles, we show two representative examples of the first convolutional layer's 32 filters [Figs. 4(a) and 4(b)] and their corresponding frequency responses [Figs. 4(c) and 4(d)], respectively. As the frequency responses highlight, these convolutional filters act as spectral band-pass filters (BPF) to extract the features shown in Fig. 2(c). When applying these two filters to the 

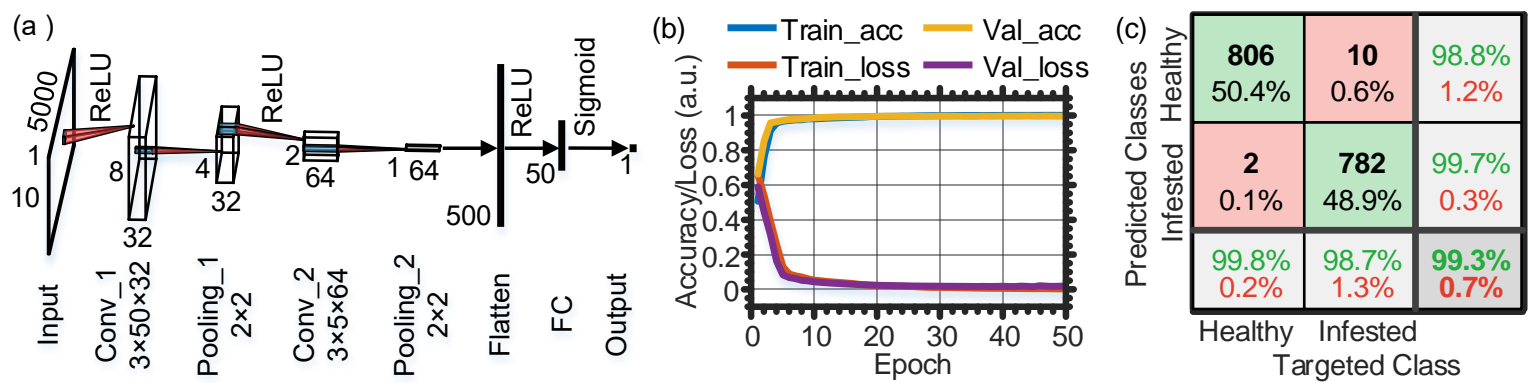

Fig. 3. Structure (a), training and validation history (b), and confusion matrix (c) of the CNN.

larvae sound / "healthy" temporal signal, the output feature maps are shown in Figs. 4(e) / 4(g) and 4(f) / 4(h), respectively. The features associated with the larvae sound comprise high-frequency components, compared with those of the "healthy" signal that may only include the low vibration frequencies produced by the wind.
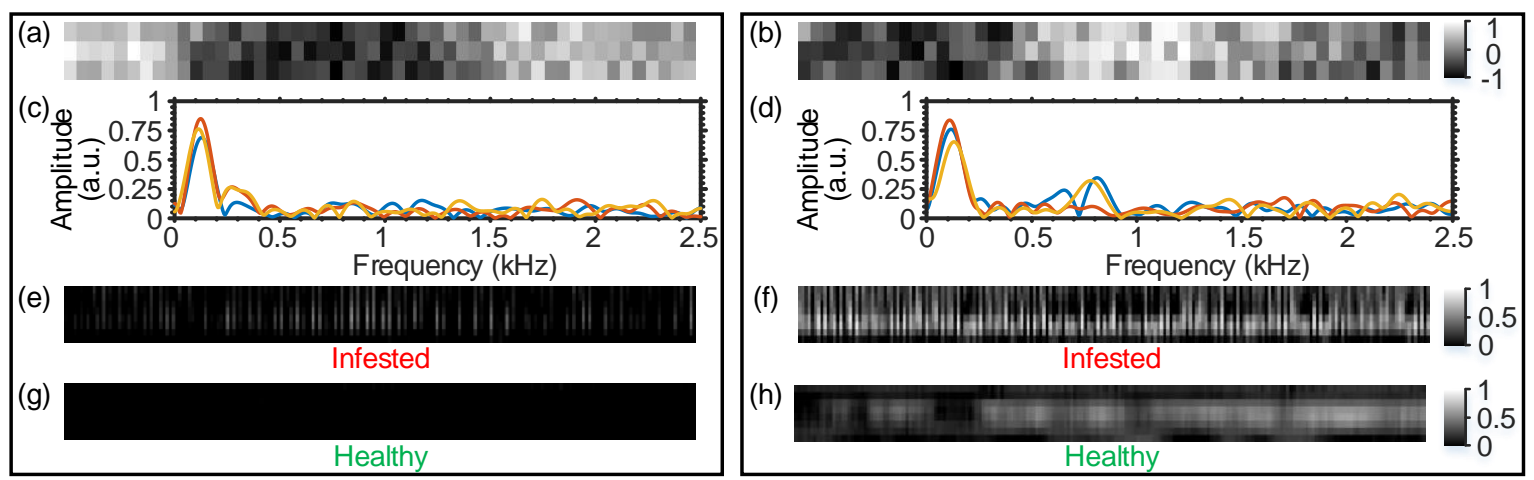

Fig. 4. Two representative examples of the first convolutional layer's filters $(a, b)$ and their corresponding frequency responses $(\mathrm{c}, \mathrm{d})$. The feature maps obtained by applying the two convolutional filters to the larvae sound (e, f) and "healthy" signal $(\mathrm{g}, \mathrm{h})$.

In summary, we employed a CNN model and a fiber optic DAS to distinguish an infested tree with RPW and a healthy tree. Within the laboratory, we reconstructed a farm's environment that included infested/healthy tree and other acoustic noise sources (wind and bird sounds). The CNN model performs perfectly with the time-domain data to offer a $99.3 \%$ classification accuracy, without the need to preprocess the data with spectral filters. This work paves the way to deploy the fiber optic DAS for the early detection of RPW in open-air and vast farms.

Acknowledgements: We acknowledge the financial support provided to this work by KAUST-Research Translation Funding (REI/1/4247-01-01).

\section{References}

1. N. M. Al-Dosary, S. Al-Dobai, and J. R. Faleiro, "Review on the management of red palm weevil rhynchophorus ferrugineus olivier in date palm phoenix dactylifera 1," Emir. J. Food Agric. pp. 34-44 (2016).

2. "Food chain crisis," http://www.fao.org/food-chain-crisis/how-we-work/ plant-protection/red-palm-weevil/en/. Accessed: 2020-12-14.

3. R. Haff and D. Slaughter, "Real-time x-ray inspection of wheat for infestation by the granary weevil, sitophilus granarius (1.)," Transactions ASAE 47, 531 (2004).

4. P. Suma, A. La Pergola, S. Longo, and V. Soroker, "The use of sniffing dogs for the detection of rhynchophorus ferrugineus," Phytoparasitica 42, 269-274 (2014).

5. I. Ashry, Y. Mao, Y. Al-Fehaid, A. Al-Shawaf, M. Al-Bagshi, S. Al-Brahim, T. K. Ng, and B. S. Ooi, "Early detection of red palm weevil using distributed optical sensor," Sci. Reports 10, 1-8 (2020).

6. I. Ashry, Y. Mao, M. S. Alias, T. K. Ng, F. Hveding, M. Arsalan, and B. S. Ooi, "Normalized differential method for improving the signal-to-noise ratio of a distributed acoustic sensor," Appl. optics 58, 4933-4938 (2019).

7. J. Schmidhuber, "Deep learning in neural networks: An overview," Neural networks 61, 85-117 (2015). 